\title{
Hypergraph Coloring Games and Voter Models
}

\author{
Fan Chung and Alexander Tsiatas
}

Abstract. We analyze a network coloring game on hypergraphs that can also describe a voter model. Each node represents a voter and is colored according to its preferred candidate (or undecided). Each hyperedge is a subset of voters that can interact and influence one another. In each round of the game, one hyperedge is chosen randomly, and the voters in the hyperedge can change their colors according to some prescribed probability distribution. We analyze this interaction model based on random walks on the associated weighted directed state graph. Under certain "memoryless" restrictions, we can use semigroup spectral methods to explicitly determine the spectrum of the state graph, and the random walk on the state graph converges to its stationary distribution in $O(m \log n)$ steps, where $n$ is the number of voters and $m$ the number of hyperedges. We can then estimate probabilities that events occur within an error bound of $\epsilon$ by simulating the voting game for $O(\log (1 / \epsilon) m \log n)$ rounds. We also consider a partially memoryless game using the memoryless game for comparison and analysis, which serves as an approximation of the actual interaction dynamics.

\section{Introduction}

We consider a network coloring game, motivated by human behavioral experiments [Kearns et al. 09, Kearns and Tan 08] conducted in a network setting.

Color versions of one or more of the figures in the article can be found online at www.tandfonline.com/uinm. 
The network coloring game can be formulated as the following interaction-based voter model.

A set of voters is modeled as the vertex set of a hypergraph $H=(V, E)$. Each hyperedge $g \in E$ represents a small, possibly overlapping group representing social interactions and discussions (such as lunchtime hallway or office discussions, blog commentary, television viewership, and Web forums). At time $t=0$, each voter has a color representing an initial preference or is undecided. Then at each time step, one hyperedge $g$ is randomly selected according to some prescribed probability distribution on $E$. After the voters in $g$ interact with one another, the voters' preferences can change probabilistically. This process is repeated for many rounds, and the coloring configuration of the voters evolves.

Many natural questions arise. Will the coloring configurations converge under certain conditions? If the coloring patterns diverge or oscillate, what would be an appropriate time frame to stop the model? When the model is run for some prescribed number of rounds, how does the observed coloring configuration behave? Can the observed coloring configuration be something other than "random"? What is the stationary distribution of the observed coloring configuration if it exists?

The behavioral experiments of [Judd and Kearns 08, Judd et al. 10, Kearns et al. 09, Kearns et al. 06] were the original motivation for our model. In these simulations, actual human agents were given a common graph-coloring task with varying monetary rewards for their timely completion. This is a strategic component that is not necessarily built into our interaction-based model, but the probability distributions for color change can potentially be derived using gametheoretic analysis. The experiments allowed agents differing "views" of information about the specific graph problem structure. Thus, agents had only limited information and could make decisions based only on the color configurations of smaller subsets of nodes (but not just pairs). This gives a compelling reason to model the voters as nodes of a hypergraph and not a traditional graph whose edges are limited to two endpoints.

Analysis of these more general voter models and coloring games have remained quite elusive in spite of extensive study either by simulation [Judd and Kearns 08, Judd et al. 10, Kearns et al. 09, Kearns et al. 06] or rigorous analysis of other proposed voter model variations [Clifford and Sudbury 73, Holley and Liggett 75, Montanari and Saberi 09, Mossel and Schoenebeck 09] for various types of networks [Tahbaz-Salehi and Jadbabaie 10]. The results vary widely with the specific set of rules or dynamics and point to evidence of intrinsic difficulties involved in the general voting model.

Although the general voting and coloring problems may be intractable to tight analysis, we will consider several special cases that will help in evaluating the 
more general case. The first is that of memoryless voter models. In a memoryless voter model, the voters do not consider their current preferences when formulating new ones. This may seem too restrictive at first, but the memoryless model lends itself to tractable mathematical analysis, and the great insight obtained provides a stepping-stone to rigorous treatment of more general models. Under these assumptions, we can show that the dynamics of coloring configurations can be analyzed quite precisely in several aspects.

Our coloring game is played on a hypergraph each hyperedge of which is formed by a group to model multiparty simultaneous interaction. Although the coloring configurations of the nodes in the hypergraph do not necessarily converge in general, we can model the rounds of coloring games by conducting random walks on the associated directed state graph, which contains coloring configurations as nodes. It turns out that for memoryless interactions, the spectrum of the state graph has elegant and well-defined expressions. Using the formula for the eigenvalues, we can determine the rate of convergence for random walks on the state graph, which can then be used for determining a cutoff time for simulating the voting game.

The eigenvectors of the random walk on the state graph contain rich information. In particular, the stationary distribution, which is the eigenvector associated with eigenvalue 1 , tells us the distribution of the observed coloring configuration as time goes to infinity. In contrast with undirected graphs, the stationary distribution of a random walk on a directed graph does not always exist and is not easy to determine, especially for state graphs with exponentially large size. Even for simple hypergraphs $H$ such as a path or cycle, the stationary distribution for random walks on the state graph has complicated forms quite different from the uniform distribution [Chung and Graham 12]. Nevertheless, we will show that events such as "red wins by at least $5 \%$ within an error bound $\epsilon$ " can be determined by simulating this voter model for $O(\log (1 / \epsilon) m \log n)$ rounds, where $m$ is the number of hyperedges in $H$, provided that the hyperedges are always chosen uniformly at random and the interactions are memoryless.

Next, we proceed to consider a partially memoryless model for which with some probability $\beta$, the interaction process is memoryless, and with probability $1-\beta$, the process is not required to be memoryless. Specifically, a partially memoryless voter model is one whose associated state-graph random walk can be decomposed into two parts $P=\beta M+(1-\beta) P^{\prime}$, where $M$ is a memoryless random walk and $P^{\prime}$ is an unrestricted random walk. Using results from the memoryless case and also from general directed Laplacians [Chung 05], we can analyze partially memoryless voter games as well.

Even the partially memoryless condition might seem to be too strict to be satisfied by real-world social interactions, but it can still be of interest to serve 
as a type of benchmark for the sake of comparison. In particular, the partially memoryless case provides a natural framework for evaluating the more general interaction voter process. For a given interaction voter process with its associated arbitrary (memory-dependent) random walk $P$, we can construct a corresponding memoryless process $P^{\prime}=\beta M+(1-\beta) P$, where $M$ is a memoryless process. The determination of $M$ depends on $\beta$ and a simulation of $P$ with an appropriate cutoff time depending on $\beta$. The details for choosing $\beta$ and $M$ will be given later, in Section 7.

\section{I.I. Related Work}

The coordination or consensus game has been extensively studied in evolutionary network game theory [Ellison 93, Kandori et al. 93, Young 93]. In a set of controlled behavioral experiments with human subjects, a simple voting game [Judd et al. 10] as well as a biased version [Kearns et al. 09] were simulated on a set of small constructed topologies. The rules varied, but they involved small financial rewards for success and differing amounts of information visibility. The results showed that consensus was often reached within a certain timeframe, though there were many cases of failure as well. It is desirable to explain these social network phenomena further with rigorous modeling and analysis.

One method to analyze various consensus games is to use the standard voter model [Clifford and Sudbury 73, Holley and Liggett 75]. In this model, there is a given network $G=(V, E)$, where the voters comprise the vertices $V$, and their social connections are represented by the edges $E$. The voter model is a Markov chain in which at each time step, one voter is selected randomly during each round; that voter chooses one of its neighbors randomly and assumes that neighbor's vote. Some empirical work has shown that its performance depends on network topology [Suchecki et al. 05]. In more rigorous analysis, several proofs have been given [Aldous and Fill 02, Yildiz et al. 10] using the dual Markov chain of coalescing random walks to show that the model results in unanimous agreement, and the expected time to reach consensus under the voter model is $O\left(n^{3} \log n\right)$, where $n$ is the number of voters in the network. For the biased voting game, this model takes exponential time [Kearns and Tan 08] to converge, but it can be used as a subroutine for an algorithm converging in $O\left(n^{8} \log n\right)$ expected time. Of course, elections in practice rarely end with a unanimous decision, and our model will reflect this observation.

Other recent work considers alternative dynamics for the voting game, including Glauber dynamics [Montanari and Saberi 09] and pieces of "advice" [Mossel and Schoenebeck 09] given to the voters. These models also result in unanimous consensus, and the time required for convergence is related to complex 
graph-theoretic quantities such as the tilted cut width, diameter, and broadcast time, often difficult to compute or reason about. Some of these models assume that the voters know approximate values for these quantities, which our model avoids. These models again result in unanimous coordination, whereas our proposed model is more likely to result in more realistic voting configurations.

The behavioral experiments of [Judd et al. 10, Kearns et al. 09] show that consensus is indeed not always reached, especially for biased voting games. However, not much has been determined thus far about the distribution of voting configurations should a true consensus be unattainable.

Furthermore, nearly all work done on the network coordination game has focused on pairwise relationships between voters. In practice, voters often interact in groups larger than two, and most of the models described so far have not taken this possibility into account. Such interactions as town-hall debate, reader comments on news articles, and dinner-table discussions can all possibly be considered in the framework of our hypergraph model.

\subsection{The Interaction Model and a Summary of Our Results}

We consider a variation on the voter model that takes into account multivoter interactions for the coordination game. Instead of limiting ourselves to pairwise relationships between voters, we model the interaction network as a hypergraph $H=(V, E)$, where each of $n$ vertices is a voter and each of $m$ hyperedges represents a group that can interact.

Our interaction model can be described as follows. At the beginning, all voters have some initial views, voting for one of several candidates, or starting out undecided. In each round, one hyperedge $g$ is selected randomly, and an interaction $X_{g, \tau}$ takes place: the randomly selected hyperedge $g$ changes its voting pattern to $\tau$. In the most general case, the probability of $X_{g, \tau}$ occurring can depend on the current coloring configuration $\sigma$ of $H$. We denote by $p(g, \sigma, \tau)$ the probability of $X_{g, \tau}$ occurring when $\sigma$ is the current coloring configuration on $H$, and for all $\sigma$, it must be the case that $\left.\sum_{g, \tau} p_{(} g, \sigma, \tau\right)=1$.

If the interactions are memoryless, then the probability of $X_{g, \tau}$ occurring is constant across all voting configurations $\sigma$ on $H$. In this case, we can omit $\sigma$ and denote by $p(g, \tau)$ the probability that $X_{g, \tau}$ occurs. When this probability has only two parameters, it can be assumed that the interaction is memoryless. The model is then simulated for some preset number of rounds.

While the game is taking place, the state can be described as a voting configuration or coloring configuration of the voters among the candidates, including "undecided." (Note that we can interpret votes as colors and vice versa, so these terms will be used interchangeably throughout this paper.) There are $r$ possible 
votes (including undecided), and thus $r^{n}$ possible configurations, and we can construct a state graph $H^{*}$, where the vertices are coloring configurations, and a directed edge connects $u$ to $v$ if the state $v$ is reachable from $u$ in one round of the interaction model. Thus, a simulation of the coloring game using the interaction model can be completely described as a random walk on the state graph $H^{*}$.

Let an event $A \subset V\left(H^{*}\right)$ be a subset of the states in the state graph. These events can represent numerous scenarios: for example, the states in which more than half of voters choose red, or the states that have a specific trend over some subsets of the voters, representing a voting district or municipality. In general, $A$ can be any event of interest. We will show that for memoryless interactions, we can estimate $\operatorname{Pr}[A]$ within a probabilistic error bound using a sufficient number of samplings, as long as $A$ contains enough states.

Theorem I.I. Let $A$ be an event, or subset of all possible coloring configurations on a hypergraph $H$ on $n$ vertices and $m$ edges. Suppose the interactions are memoryless, the probability of selecting a hyperedge $g$ in any given round is at least $1 /(\alpha m)$ for some $\alpha$, and for every set $S \subseteq V(H)$, there are at least $|S|$ edges incident to vertices in $H$. The probability $\operatorname{Pr}[A]$ that $A$ occurs at any cutoff time after $O(\alpha m \log n)$ rounds of simulation can be estimated within an error bound of $\epsilon$ using

$$
O\left(\frac{\alpha \log (1 / \epsilon) m \log (n)}{\operatorname{Pr}[A]}\right)
$$

rounds of simulation.

Note that for the special case that the hyperedges are chosen uniformly at random, the number of rounds of simulation for convergence is

$$
O\left(\frac{\log (1 / \epsilon) m \log (n)}{\operatorname{Pr}[A]}\right) \text {. }
$$

The main tools that we use to prove the above theorem are sampling and fast mixing of random walks on the state graph $H^{*}$ associated with memoryless strategies. In particular, the spectrum of such random walks on the state graph $H^{*}$ can be determined using spectral techniques originating in the analysis of card shuffling [Brown 00, Brown and Diaconis 98], self-organizing search [Fill 96], hyperplane arrangement [Bidigare et al. 99], and semigroup random walks as well as the recent work on edge-flipping games in graphs [Chung and Graham 12].

For the partially memoryless case, in which the random walk $P$ on the state graph $H^{*}$ can be written as $P=\beta M+(1-\beta) P^{\prime}$ with memoryless $M$, we have the following result showing a tradeoff between the convergence time and $\beta$ : 
Theorem 1.2. Let $A$ be an event or subset of all possible coloring configurations on a hypergraph $H$ on $n$ vertices and $m$ edges. Suppose the interactions are partially memoryless with parameter $\beta$, the probability of selecting a hyperedge $g$ in any given round is at least $1 /(\alpha m)$ for some $\alpha$, and for every set $S \subseteq V(H)$, there are at least $|S|$ edges incident to vertices in $H$. The probability $\operatorname{Pr}[A]$ that $A$ occurs at any cutoff time after $O(\alpha m \log n)$ rounds of simulation can be estimated within an error bound of $\epsilon$ using

$$
O\left(\frac{\alpha \log (1 / \epsilon) m \log n}{\beta \operatorname{Pr}[A]}\right)
$$

rounds of simulation.

Finally, the most general case, in which the interactions are not memoryless at all, is well known to be very difficult to analyze exactly [Chung and Graham 12]. This is because the associated random walk can have exponentially small eigenvalues, and convergence time can vary widely based on the specific dynamics. Nevertheless, we will show how to use insight from the memoryless and partially memoryless models to reason about the general voter model. By choosing an appropriate damping constant $\beta$ and extracting a memoryless version of a given process, we can then construct a partially memoryless process that can be used to approximate the given interactive process.

\section{The Voting Game on a Hypergraph as a Random Walk on the Associated State Graph}

As stated in the previous section, the interaction model on a hypergraph $H=$ $(V, E)$ can be analyzed as a random walk on a state graph $H^{*}$. The state graph $H^{*}$ is a weighted directed graph whose vertices are coloring configurations of $V(H)$. To distinguish from nodes in $H$, we sometimes call a vertex in $V\left(H^{*}\right)$ a state. There is a directed edge from a state $u$ in $H^{*}$ to another state $v$ if there exist a hyperedge $g$ and a coloring pattern $\tau$ on $g$ such that the interaction $X_{g, \tau}$ moves $u$ to $v$. The weight on the edge $(u, v)$ is determined by the probability that $X_{g, \tau}$ occurs during state $u$. We denote by $p(g, u, \tau)$ the probability that in state $u$, hyperedge $g$ is selected and changes its color configuration to $\tau$.

Note that for a directed graph with weighted edges, we can define a typical random walk with a transition from $u$ to $v$ occurring with probability $w(u, v) / \sum_{z} w(u, z)$. Therefore, the typical random walk associated with the state graph $H^{*}$ simulates the evolving configurations in the voter interaction game. 
Starting from a coloring configuration $u$, a sequence of interactions

$$
X_{g_{1}, \tau_{1}} X_{g_{2}, \tau_{2}} \ldots X_{g_{t}, \tau_{t}}
$$

induces a series of changes in the coloring configuration and can then be viewed as a walk starting from $u$ and traversing $t$ directed edges on the state graph $H^{*}$. This correspondence leads to the following lemma, whose proof follows from the Perron-Frobenius theorem.

Lemma 2.I. The voter interaction game with interactions as described above does not converge to an equilibrium in general. Instead, from any initial coloring configuration, the resulting configuration after $t$ rounds of simulation is $s$ with probability approaching $\pi(s)$, where $\pi$ is the stationary distribution of the random walk on the state graph $H^{*}$, as long as $t$ is sufficiently large and $H^{*}$ is strongly connected and aperiodic.

An interaction $X_{g, \tau}$ is said to be nontrivial if the associated probability $p(g, \sigma, \tau)$ is nonzero. In the special case that all nontrivial strategies are consistent with some coloring pattern $\tau$ (for example, all red), then the coloring configuration will reach an equilibrium. (Note that this is an example of the special case in which the interactions are memoryless.) Suppose there is a state $s$ for which all the nontrivial interactions are of the form $X_{g, s_{g}}$, where $s_{g}$ is simply the coloring configuration of $s$ restricted to nodes in $g$. If this is the case, then starting from any initial configuration, the voting game will converge using standard coupon-collector probabilistic arguments.

Lemma 2.2. In the voter interaction game, suppose there exists a coloring configuration s such that all the nontrivial interactions are of the form $X_{g, s_{g}}$, where $s_{g}$ denotes the coloring pattern of $s$ restricted to voters in $g$. Starting from any initial configuration, the voting game converges to $s$ after $t$ rounds of simulation with probability at least $1-e^{-c}$ if

$$
t \geq \frac{\log n+c}{\min _{v \in V} \sum_{\substack{g \in E \\ g \ni v}} p\left(g, s_{g}\right)},
$$

where $n$ is the number of voters and $p\left(g, s_{g}\right)$ is the probability associated with the interaction. In the case that every vertex is incident to exactly d hyperedges and each hyperedge is chosen with equal probability, the above inequality is just $t \geq n(\log n+c)$. 
For the remainder of this paper, we will assume that the given hypergraph and interaction dynamics yield a state graph $H^{*}$ that is aperiodic and strongly connected. We will refer to the stationary distribution $\pi$ accordingly.

We remark that Lemma 2.1 reduces the voter interaction game to the study of the associated random walk on the state graph, and the rate of convergence depends on the eigenvalues of the directed state graph. These values can be complex, and in the most general case, the spectral gap can be exponentially small. Nevertheless, we will consider memoryless interactions that allow us to have real eigenvalues for the state graph, and we can use these techniques to derive some bounds for partially memoryless interactions as well.

\section{Memoryless Interactions and Semigroup Spectral Graph Theory}

For a random walk on the state graph $H^{*}$, we can describe a path in terms of the interactions $\left\{X_{g, \tau}\right\}$ that take place to follow the path. Thus, it is convenient to describe random walks as sequences of interactions. For a sequence $S=X_{g_{1}, \tau_{1}} X_{g_{2}, \tau_{2}} \ldots X_{g_{t}, \tau_{t}}$ and a state $u$, we say that $S=u$ if the interaction game leads to state $u$ after following the path described by $S$. For two sequences, we say that $S_{1}=S_{2}$ if both paths end at the same state.

We say that the nontrivial interactions $\left\{X_{g, \tau}\right\}$ are memoryless if the probability of choosing them does not depend on the current state. An equivalent definition is that for memoryless interactions, a repeated interaction $X_{g, \tau}$ means that earlier occurrences have no effect. Namely, for any three sequences of interactions $S_{1}, S_{2}, S_{3}$ of any length, then

$$
S_{1} X_{g, \tau} S_{2} X_{g, \tau} S_{3}=S_{1} X_{g, \tau} S_{2} S_{3}
$$

If the interaction strategies are memoryless, we can view them as members of a special type of semigroup known as a left-regular band, or LRB, first studied in the 1940s [Klein-Barmen 40, Schützenberger 47]. An LRB is a semigroup every element of which is idempotent, and for any two elements $x, y \in S$, one has $x y x=x y$. We define the product of two interactions $X_{g, \tau}$ and $X_{g^{\prime}, \tau^{\prime}}$ to be the two interactions in sequence. If the interactions are memoryless, it is easy to see that the semigroup $S$ generated by all nontrivial interactions $X_{g, \tau}$ is an LRB. This allows us to apply techniques in [Bidigare et al. 99, Brown 00, Brown and Diaconis 98, Chung and Graham 12] to the voter interaction game. In particular, the associated random walk on the state graph for memoryless interactions has a clean form, as stated in the following theorem. 
Theorem 3.I. Suppose that the voter interaction game on a hypergraph $H=(V, E)$ in $r$ colors has memoryless interactions $X_{g, \tau}$ for $g \in E$ and coloring patterns $\tau$ on $g$. If $p(g, \tau)$ is the probability of choosing $g$ and coloring voters in $g$ with the coloring pattern $\tau$, then the random walk on the associated state graph $H^{*}$ has an eigenvalue $\lambda_{T}$ for every subset $T \subseteq V$ :

$$
\lambda_{T}=\sum_{\substack{g, \tau \\ g \subseteq T}} p(g, \tau),
$$

with multiplicity $(r-1)^{n-|T|}$.

For the specific case in which the hyperedges are selected uniformly at random and there are only two colors, we note in the following corollary that the eigenvalues have an even cleaner form.

Corollary 3.2. For each subset $T \subseteq V$, there is an eigenvalue

$$
\lambda_{T}=\frac{|\{g \in E \mid g \subseteq T\}|}{m}
$$

with multiplicity 1.

To prove Theorem 3.1, we need to explore further properties of LRB semigroups. The proof of the corollary follows from [Chung and Graham 12, Theorem A], and the theorem follows by generalizing the techniques to $r$ colors. But in order to use these results, we must first interpret semigroup terminology in terms of the voter interaction game.

For any LRB semigroup $S$, there is a natural partial order defined by $x \leq$ $y \Leftrightarrow x y=y$. For our LRB, whose members are sequences of interactions $X_{g, \tau}$, we define $x \leq y$ if the interaction or sequence of interactions represented by $x$ is irrelevant after the sequence $y$ is performed. A semilattice $L(S)$ can be defined on $S$ by considering the relation $\preceq$ on $S$ as follows: $y \preceq x \Leftrightarrow x y=x$. In other words, $y \preceq x$ if for two sequences of interactions $x$ and $y$, the concatenation $x y$ leads to the same state as $x$ by itself. The equivalence class under $\preceq$ that contains $x$ is said to be the support of $x$, denoted by $\operatorname{supp}(x)$, and for $x, y \in S$, $\operatorname{supp}(x y)=\operatorname{supp}(x) \cup \operatorname{supp}(y)$. The support of $x$ can be interpreted as the set of vertices whose colors were affected by the sequence of interactions given by $x$. The various elements of $L(S)$ are called the flats, and an element of $S$ is said to be a chamber if its support is maximal. Therefore, the chambers of $S$ are simply sequences of interactions $x$ that affect the entire set of nodes $V$.

As given in [Brown 00, Brown and Diaconis 98, Chung and Graham 12], the eigenvalues of a random walk on chambers have an elegant form. For each flat 
$X \in L(S)$, there is an eigenvalue $\lambda_{X}=\sum_{x \in X} w_{x}$. Here, $w_{x}$ is the probability of selecting the semigroup member $x$. In the voter interaction model, the chambers represent coloring configurations, and the probabilities $w_{x}$ are simply the probabilities of choosing specific interactions, which are constant in the memoryless case. The flats are simply subsets of $V$. The theorem statement about the eigenvalues of the random walk on $H^{*}$ follows from this interpretation of the flats of the LRB semigroup induced by the memoryless interactions. For the multiplicities, we note from [Chung and Graham 12] that the multiplicity of $m_{X}$ of $\lambda_{X}$ satisfies

$$
\sum_{Y \succeq X} m_{Y}=c_{X}
$$

where $c_{Y}$ is the cardinality of $S_{\geq Y}=S_{\geq y}=\{z \in S: z \geq y\}$, where $y$ is any element with support $Y$. (The cardinality is independent of the choice of $y$.) Translating the semigroup terminology into the setting of the interaction model shows how the multiplicities given in Theorem 3.1 were derived.

We note that these techniques as used in [Chung and Graham 12] were developed for an edge-flipping game with two colors. The semigroup techniques used generalize to $r$ colors, and the full proof of Theorem 3.1 can be derived by this simple extension. Further details about LRB semigroups and their terminology can be found in [Brown 00].

\section{The Cutoff Time for Voter Interaction Games}

Our methods also address some of the questions that have arisen in the recent human network experiments of [Kearns and Tan 08]. The voters are given a hard deadline, often arbitrarily set by various entities without rigorous justification. Furthermore, for different stopping times, the outcome of the network experiments varied widely from consensus to chaos. Using our interaction model and spectral techniques, we will prove the following theorems about the interaction model's convergence properties, as well as a mathematical interpretation of the resulting voting configuration after convergence is reached.

For voter interaction games with memoryless interactions, we have the following theorem. The proof uses the spectrum of $H^{*}$, as derived in the previous section, to bound the total variation distance between the random walk and its stationary distribution after $t$ steps.

Theorem 4.I. Suppose the interaction model is simulated on a hypergraph $H=$ $(V, E)$ with $|V|=n$, and each voter in $V$ is colored with one of $r$ colors. If 
the interactions are memoryless, then the total variation distance between the random walk on the state graph $H^{*}$, denoted by the transition probability matrix $P$ after $t$ rounds of simulation and its stationary distribution $\pi$, is given by

$$
\left\|P^{t}-\pi\right\|_{T V}=\max _{A \subseteq V} \max _{y}\left|\sum_{x \in A} P^{t}(y, x)-\pi(x)\right| \leq \sum_{T \subseteq H} \lambda_{T}^{t}(r-1)^{n-|T|} .
$$

We remark that the above bound can be somewhat improved by restricting $T$ to be the comaximal subsets, although asymptotically, the bound is still the same. Using this bound on the total variation distance, we can derive the convergence time for the interaction model:

Theorem 4.2. On a hypergraph $H=(V, E)$ with $|V|=n,|E|=m$, suppose the voter interaction model is simulated with memoryless interactions, and the probability of choosing a hyperedge $g$ in any round is uniform over E. Suppose that for every set $S$ of $k$ voters, there are at least $k$ hyperedges involving voters in $S$. The random walk on the state graph $H^{*}$ converges to its stationary distribution in $O(m \log n)$ steps.

To prove Theorem 4.2, we use the derived spectrum from Theorem 3.1 in our derived bound on the total variation distance from Theorem 4.1.

Proof. Using Theorems 3.1 and 4.1, we obtain

$$
\begin{aligned}
\left\|P^{t}-\pi\right\|_{T V} & \leq \sum_{T \subseteq H} \lambda_{T}^{t}(r-1)^{n-|T|} \\
& =\sum_{T \subseteq V}\left(\frac{|\{g \in E \mid g \subseteq T\}|}{m}\right)^{t}(r-1)^{n-|T|} \\
& \leq \sum_{k=1}^{n}\left(\frac{\max _{|T|=k}|\{g \in E \mid g \subseteq T\}|}{m}\right)^{t}\left(\begin{array}{l}
n \\
k
\end{array}\right)(r-1)^{n-k} .
\end{aligned}
$$

Here, we indexed the subsets $T \subseteq V$ by their sizes.

For every node set $T$ of size $k$, we can bound the number of hyperedges contained within $T$ from above. Using the fact that for every set $T$ with $|T|=k$, there are at least $k$ hyperedges incident to nodes in $T$, we have

$$
\left\|P^{t}-\pi\right\|_{T V} \leq \sum_{k=1}^{n}\left(1-\frac{k}{m}\right)^{t}\left(\begin{array}{l}
n \\
k
\end{array}\right) 2^{n-k} \leq n^{2}\left(1-\frac{1}{m}\right)^{t} \leq e^{-c},
$$


since for

$$
f(k)=\left(1-\frac{k}{m}\right)^{t}\left(\begin{array}{l}
n \\
k
\end{array}\right) 2^{n-k},
$$

we have $f(k) \geq f(k+1)$ for $k \geq 1$ and $t>2 m \log n+c m$. The theorem is proved.

Theorems 4.1 and 4.2 imply a method for choosing a stopping point for the interaction model: enough time for a desired level of convergence to a stationary distribution $\pi$. This also indicates what the voting configuration among the agents looks like at any time after it has converged: the votes are a random sample from $\pi$ from all the voting configurations in the state graph.

\section{Estimating the Expected Value of a Given Event}

Although estimating individual components of $\pi$ can be computationally intractable, for memoryless interactions, we can effectively use sampling to estimate the probability of an event $A$, as long as the event has enough probability mass. For a general $\pi$, it can be difficult to reason about its components, since it contains exponentially many elements, corresponding to the state graph. Even for simple graphs such as the path of length $k$, the exact stationary distribution on the state graph is quite complex [Chung and Graham 12]. Additionally, because most of the components of $\pi$ are exponentially small, even estimating $\pi$ can be quite difficult. But in practice, the exact stationary distribution is not of utmost importance. Instead, it is much more revealing and tractable to reason about events that capture a larger portion of $\pi$. We will use the following fact.

Theorem 5.I. [Motwani and Raghavan 95] Let $A$ be an event and $\pi(A)=\sum_{s \in A} \pi(s)$ the probability that the outcome is in $A$. Let $\delta, \epsilon \in(0,1)$. Suppose that after $N$ samplings, $X$ is the proportion of times the outcome was in $A$. Then

$$
\operatorname{Pr}[(1-\delta) \pi(A) \leq X \leq(1+\delta) \pi(A)] \leq 1-\epsilon,
$$

as long as

$$
N \geq O\left(\frac{\log (1 / \epsilon)}{\pi(A) c(\delta)}\right),
$$

where $c(\delta)$ depends only on $\delta$. 
We can use this bound to prove Theorem 1.1. Here, we consider the case that each hyperedge is chosen with uniform probability $1 / m$, but the same argument will hold using the looser $1 /(\alpha m)$ bound.

Proof of Theorem I.I. For an event $A$, suppose we are given an initial state $f$, which we denote by a row vector indexed by states in $V\left(H^{*}\right)$. For every integer $t$, the coloring configuration we observe after $t$ rounds of the voter interaction model is in $A$ with probability

$$
\mathrm{E}_{t}[f A]=\sum_{x \in A} f P^{t}(x)
$$

where $P$ denotes the transition probability matrix of the random walk on $H^{*}$.

By combining Theorems 3.1 and 4.1, we have

$$
\begin{aligned}
\left|\mathrm{E}_{t}[A]-\pi(A)\right| & \leq \max _{y}\left|\sum_{x \in A} P^{t}(y, x)-\pi(x)\right|=\left\|P^{t}-\pi\right\|_{T V} \\
& \leq \sum_{T \subseteq V} \lambda_{T}^{t}(r-1)^{n-|T|} .
\end{aligned}
$$

For the case that each hyperedge is chosen with probability $1 / m$, where $m$ is the total number of hyperedges, we have

$$
\left|\mathrm{E}_{t}[A]-\pi(A)\right| \leq n^{2}\left(1-\frac{1}{m}\right)^{t} \leq \epsilon
$$

if $t>2 m(\log n+\log (1 / \epsilon))$.

Now we use Theorem 5.1 by breaking up the voter interaction game into $N$ phases, where

$$
N=O\left(\frac{\log (1 / \epsilon)}{\pi(A) c(\delta)}\right),
$$

and each phase consists of $t=O(m \log n)$ rounds. The proportion of phases in which the outcome is in $A$ satisfies

$$
\operatorname{Pr}[(1-\delta) \pi(A)<|X-\pi(A)|]<(1+\delta) \pi(A) \geq 1-2 \epsilon
$$

\section{The Interaction Model with Partially Memoryless Interactions}

We say that a set of interactions is partially memoryless if the random walk on the state graph $H^{*}$ can be decomposed into two parts, one of which is memoryless. 
Specifically, if the random walk transition matrix is $P$, there is a $\beta \in(0,1)$ such that we can write

$$
P=\beta M+(1-\beta) P^{\prime}
$$

where the interactions described by $M$ are memoryless and $P^{\prime}$ is another transition probability matrix without any restrictions.

One way in which a partially memoryless interaction model can arise is if at each step, the voters in the selected hyperedge interact without memory with some probability $\beta$, allowing their actions to depend on the current state with probability $1-\beta$. It is also possible that the transition probability matrix $P^{\prime}$ is not explicitly built this way, but it can nevertheless be expressed as such a partially memoryless model. In this sense, the parameter $\beta$ can be viewed as describing how memoryless the model is, and its properties will depend on $\beta$.

Theorem 6.I. Suppose the voter interaction model is simulated with partially memoryless interactions on a hypergraph $H=(V, E)$ with $|V|=n,|E|=m$, and the probability of choosing a hyperedge $g$ in any round is uniform over E. Suppose that for every set $S$ of $k$ voters, there are at least $k$ hyperedges involving voters in $S$. The random walk on the state graph $H^{*}$ converges to its stationary distribution in $O(m \log n / \beta)$ steps.

The partially memoryless structure allows us to use the results from the fully memoryless case, with an additional factor of $1 / \beta$ when the spectrum is analyzed.

Proof. Because the random walk on $H^{*}$ is given by memoryless strategies, we can write its transition matrix as $\beta P_{1}+(1-\beta) P_{2}$ for memoryless $P_{1}$. Theorem 3.1 allows us to analyze the eigenvalues of $P_{1}$; we can use results from [Chung 05] to see that $P_{2}$ has all eigenvalues between 0 and 1 . Thus, if $\lambda_{T}$ is an eigenvalue of $P_{1}$, then there is a corresponding eigenvalue of $P=\beta P_{1}+(1-\beta) P_{2}$ satisfying

$$
\lambda \leq \beta \lambda_{T}+(1-\beta) .
$$

Using Theorem 4.1, we have

$$
\begin{aligned}
\left|\mathrm{E}_{t}[A]-\pi(A)\right| & \leq \max _{y}\left|\sum_{x \in A} P^{t}(y, x)-\pi(x)\right|=\left\|P^{t}-\pi\right\|_{T V} \\
& \leq \sum_{T \subseteq V} \lambda_{T}^{t}(r-1)^{n-|T|} \leq \sum_{k=1}^{n}\left(1-\frac{\beta k}{m}\right)^{t}\left(\begin{array}{l}
n \\
k
\end{array}\right)(r-1)^{n-k} \\
& \leq n^{2}\left(1-\frac{\beta}{m}\right)^{t} \leq e^{-c}
\end{aligned}
$$


if

$$
t \geq \frac{2 m \log n+c n}{\beta}
$$

With this result, we can prove Theorem 1.2.

Proof of Theorem 1.2. For an event $A$, suppose we are given an initial state $f$, which we denote by a row vector indexed by states in $V\left(H^{*}\right)$. For an integer $t$, the coloring configuration we observe after $t$ rounds of the voter interaction model is in $A$ with probability

$$
\mathrm{E}_{t}[f A]=\sum_{x \in A} f P^{t}(x),
$$

where $P$ denotes the transition probability matrix of the random walk on $H^{*}$.

By combining Theorems 3.1 and 4.1, we have

$$
\begin{aligned}
\left|\mathrm{E}_{t}[A]-\pi(A)\right| & \leq \max _{y}\left|\sum_{x \in A} P^{t}(y, x)-\pi(x)\right|=\left\|P^{t}-\pi\right\|_{T V} \\
& \leq \sum_{T \subseteq V} \lambda_{T}^{t}(r-1)^{n-|T|} .
\end{aligned}
$$

For the case that each hyperedge is chosen with probability $1 / m$, where $m$ is the total number of hyperedges, we have

$$
\left|\mathrm{E}_{t}[A]-\pi(A)\right| \leq n^{2}\left(1-\frac{\beta}{m}\right)^{t} \leq \epsilon
$$

if

$$
t>\frac{m}{\beta}\left(2 \log n+\log \left(\frac{1}{\epsilon}\right)\right) .
$$

Now we use Theorem 5.1, similarly to how it was used in the proof of Theorem 1.1, by breaking up the voter interaction game into $N$ phases, where

$$
N=O\left(\frac{\log (1 / \epsilon)}{\pi(A) c(\delta)}\right)
$$

and each phase consists of $t=O(m \log n / \beta)$ rounds, from (6.1). We have the same bound as (5.1); the proportion of phases in which the outcome is in $A$ satisfies

$$
\operatorname{Pr}[(1-\delta) \pi(A)<|X-\pi(A)|]<(1+\delta) \pi(A)] \geq 1-2 \epsilon
$$




\section{The General Interaction Model}

Thus far, we have written about the interaction model with memoryless and partially memoryless interactions. The general version of the interaction model concerns interactions all of which can depend on the current and previous states, and the asymptotic properties of a random walk on the state graph $H^{*}$ can be quite difficult to analyze. The eigenvalues of the random walk can be real or complex, and exponentially small [Chung and Graham 12]. With $O\left(2^{n}\right)$ states, computing the spectrum explicitly is too expensive or infeasible. It is even too difficult just to estimate the eigenvalues. Nevertheless, we can use the previous memoryless and partially memoryless models as a basis for comparison between a specific set of state-dependent interactions and memorylessness in general.

In particular, for any general interaction voter game, we can build a companion game that is partially memoryless with one scalar parameter $\beta$. Let $M$ denote the transition probability matrix of the random walk on $H^{*}$. We wish to construct a partially memoryless model

$$
P^{\prime}=\beta M+(1-\beta) P,
$$

where $M$ is memoryless and can be constructed from $P$ and $\beta$ by simulation as follows.

The main idea is to simulate the general model $M$ for some number of rounds, building a memoryless model using the simulation outcome. To know how long to simulate, we refer to Theorem 1.2, which provides an upper bound on the convergence time to the stationary distribution for partially memoryless interactions. If the companion model serves as an approximation for the actual voter game, it is enough to simulate the more general model in $O(m \log n / \beta)$ time. To construct $M$, we collect a sample probability distribution: when a hyperedge $g$ is selected, keep track of the resulting coloring configurations. Then we can use these samples to build a partially memoryless model: first select a hyperedge $g$; then with probability $\beta$, randomly select one of the collected sample coloring configurations on $g$. Note that once the samples have been collected, this step is memoryless. With probability $1-\beta$, we just use the original memory-dependent dynamics.

The problem of determining the appropriate value of $\beta$ can be quite difficult, since the required length of time until convergence may be exponential. A feasible heuristic approach is a combination of a series of iteration and simulation. Such a process also can be used to get a sense of how "memoryless" the more general model really is. In other words, an alternative interpretation of the damping constant $\beta$ is just the ratio of the rate of convergence of the memoryless random walk and the actual random walk. In general, the value of $\beta$ can range from 1 


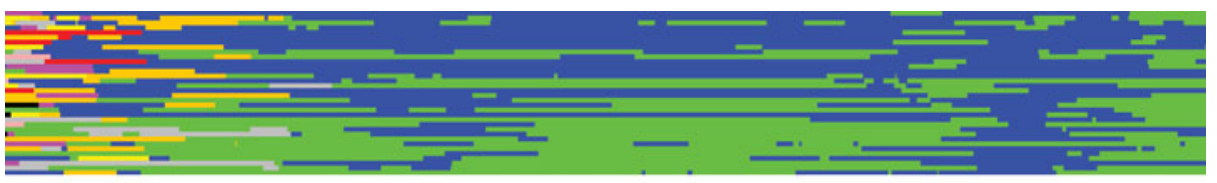

(a) One simulation of the consensus game on Zachary's karate network for 1000 steps.

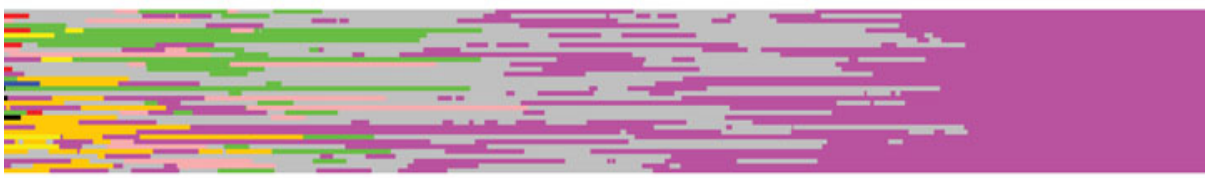

(b) Another simulation of the consensus game on Zachary's karate network for 1000 steps.

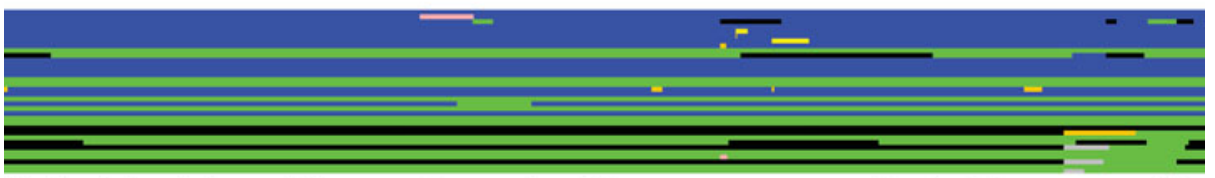

(c) Simulation of the partially memoryless version of the consensus game on Zachary's karate network for 1000 steps, with $\beta=0.01$ and 100 steps of training.

Figure I. Simulations of the consensus game and its partially memoryless approximation.

to some exponentially small values, reflecting the fact that some models have a higher extent of memorylessness than others. So even though the general model is notoriously difficult to reason about, we can still quantify its convergence time empirically by comparing with the partially memoryless model.

To illustrate this comparative process, we consider a traditional voting game on networks. We are given a graph $G$, and each node has a starting color. Then at each time step, a node is randomly selected, and it takes the color of one of its neighbors, selected uniformly at random. In our setting, there is a hyperedge for every node $v$, consisting of $v$ and its neighbors, and whenever it is chosen, $v$ changes color to one of its neighbors' colors. It should be clear that these dynamics are completely memory-dependent: the coloring configuration at time $t+1$ always depends on the state at time $t$. But we can build a partially memoryless process using the method described in the previous paragraph.

We demonstrate this method using Zachary's karate network [Zachary 77] as an example graph. This graph has 34 nodes, and we initially assign one of nine colors randomly to each node. Two sample runs of the consensus game are shown in Figures 1(a) and 1(b). Each row represents one node, and the colors change as time moves from left to right.

We note that the state graph can be as large as $9\left(\begin{array}{c}34 \\ 2\end{array}\right)$ nodes. The convergence bound for a memoryless game of similar size is about $O(m \log n)$ steps with 
$n=34$ and $m=\left(\begin{array}{c}34 \\ 2\end{array}\right)$. This is comparable to the time limit of 1000 steps if each step is taken to be in the range of a fraction of a second. But in our simulations, consensus may or may not be reached.

In Figure 1(c), we give an illustration of a partially memoryless version of the consensus game. Using only 100 rounds of simulation, we built the partially memoryless version and simulated it for 1000 rounds. In practice, $\beta$ can be chosen empirically using binary search on $(0,1)$. For Figure $1(\mathrm{c})$, we chose $\beta=0.01$. One way to choose $\beta$ is by iteratively adjusting its value so that the proportion of cases that achieve consensus reaches the range of what is to be expected.

Funding. Fan Chung's research was supported in part by ONR MURI N000140810747 and AF/SUB 552082.

\section{References}

[Aldous and Fill 02] D. Aldous and J. A. Fill. Reversible Markov Chains and Random Walks on Graphs. University of California, Berkeley, 2002.

[Bidigare et al. 99] T. P. Bidigare, P. Hanlon, and D. N. Rockmore. "A Combinatorial Description of the Spectrum for the Tsetlin Library and Its Generalization to Hyperplane Arrangements." Duke Mathematical Journal 99 (1999), 135-174.

[Brown 00] K. S. Brown. "Semigroups, Rings, and Markov Chains." Journal of Theoretical Probability 13 (2000), 837-938.

[Brown and Diaconis 98] K. S. Brown and P. Diaconis. "Random Walks and Hyperplane Arrangements." Annals of Probability 26 (1998), 1813-1854.

[Chung 05] F. Chung. "Laplacians and the Cheeger Inequality for Directed Graphs." Annals of Combinatorics 9 (2005), 1-19.

[Chung and Graham 12] F. Chung and R. Graham. "Edge Flipping in Graphs." Advances in Applied Mathematics 48 (2012), 37-63.

[Clifford and Sudbury 73] P. Clifford and A. Sudbury. "A Model for Spatial Conflict." Biometrika 60 (1973), 581-588.

[Ellison 93] G. Ellison. "Learning, Local Interaction, and Coordination." Econometrica 61 (1993), 1047-1071.

[Fill 96] J. A. Fill. "An Exact Formula for the Move-to-Front Rule for Self-Organizing Lists." Journal of Theoretical Probability 9 (1996), 113-160.

[Holley and Liggett 75] R. A. Holley and T. M. Liggett. "Ergodic Theorems for Weakly Interacting Infinite Systems and the Voter Model." Annals of Probability 3 (1975), 643-663.

[Judd and Kearns 08] S. Judd and M. Kearns. "Behavioral Experiments in Networked Trade." In Proceedings of the 9th ACM Conference on Electronic Commerce, pp. 150$159,2008$. 
[Judd et al. 10] S. Judd, M. Kearns, and Y. Vorobeychik. "Behavioral Dynamics and Influence in Networked Coloring and Consensus." Proceedings of the National Academy of Sciences 107 (2010), 14978-14982.

[Kandori et al. 93] M. Kandori, G. Mailath, and R. Rob. "Learning, Mutation, and Long Run Equilibria in Games." Econometrica 61 (1993), 29-56.

[Kearns and Tan 08] M. Kearns and J. Tan. "Biased Voting and the Democratic Primary Problem." In Proceedings of the 4th International Workshop on Internet and Network Economics (WINE ), pp. 639-652, 2008.

[Kearns et al. 06] M. Kearns, S. Suri and N. Montfort. "An Experimental Study of the Coloring Problem on Human Subject Networks." Science 313 (2006), 824-827.

[Kearns et al. 09] M. Kearns, S. Judd, T. Tan, and J. Wortman. "Behavioral Experiments on Biased Voting in Networks." Proceedings of the National Academy of Sciences 106 (2009), 1347-1352.

[Klein-Barmen 40] F. Klein-Barmen. "Über eine weitere Verallgemeinerung des Verbandsbegriffes." Mathematische Zeitschrift 46 (1940), 472-480.

[Montanari and Saberi 09] A. Montanari and A. Saberi. "Convergence to Equilibrium in Local Interaction Games and Ising Models." ACM SIGecom Exchanges 8 (2009), $1-4$.

[Mossel and Schoenebeck 09] E. Mossel and G. Schoenebeck. "Reaching Consensus on Social Networks." In Proceedings of the First Symposium on Innovations in Computer Science (ICS 2009), pp. 214-229, 2009.

[Motwani and Raghavan 95] R. Motwani and P. Raghavan. Randomized Algorithms. Cambridge University Press, 1995.

[Schützenberger 47] M.-P. Schützenberger. "Sur certains treillis gauches." Comptes Rendus de l'Académie des Sciences 224 (1947), 777-778.

[Suchecki et al. 05] K. Suchecki, V. Eguíluz, and M. San Miguel. "Voter Model Dynamics in Complex Networks: Role of Dimensionality, Disorder, and Degree Distribution." Physical Review E 72 (2005), 1-8.

[Tahbaz-Salehi and Jadbabaie 10] A. Tahbaz-Salehi and A. Jadbabaie. "Consensus over Ergodic Stationary Graph Processes." IEEE Transactions on Automatic Control 55 (2010), 225-230.

[Yildiz et al. 10] M. Yildiz, R. Pagliari, A. Ozdaglar, and A. Scaglione. "Voting Models in Random Networks." In Proceedings of the 2010 Information Theory and Applications Workshop (ITA), pp. 1-7, 2010.

[Young 93] H. P. Young. "The Evolution of Conventions." Econometrica 61 (1993), $57-84$.

[Zachary 77] W. W. Zachary. "An Information Flow Model for Conflict and Fission in Small Groups." Journal of Anthropological Research 33 (1977), 452-473. 
Fan Chung, Department of Computer Science and Engineering, University of California, San Diego, 9500 Gilman Drive, La Jolla, CA 92093-0404, USA (fan@cs.ucsd.edu)

Alexander Tsiatas, Department of Computer Science and Engineering, University of California, San Diego, 9500 Gilman Drive, La Jolla, CA 92093-0404, USA (atsiatas@ gmail.com) 